\section{The Dilemma of Central Serous Retinopathy, a Corticosteroid-induced Complication, in Patients with Ocular Inflammatory Disease}

\section{To the Editor:}

Rheumatologists sometimes collaborate with ophthalmologists in the management of patients with ocular inflammatory disease. Corticosteroids are often a valuable component of this management. Ophthalmic complications associated with corticosteroid usage include cataract and glaucoma. Corticosteroids have also been implicated in another important cause of visual loss, central serous retinopathy $(\mathrm{CSR})^{1,2}$, a disorder characterized by neurosensory detachment of the retina affecting the macula.

The combination of CSR, best managed by reduction in corticosteroid dosage, and inflammation usually requiring increased corticosteroid dosage creates a dilemma such that worsening visual acuity should be treated paradoxically with reduction in corticosteroid dosage.

Case report 1. A 34-year-old male patient with history of juvenile idiopathic arthritis and uveitis complained of blurred vision in the left eye for 3 days, which was 3 weeks after increasing prednisone dose to $10 \mathrm{mg}$ daily. He was functionally blind in the right eye due to band keratopathy. Vision was $20 / 25+2$ in the left eye. The fundus examination revealed CSR, confirmed by ocular coherence tomography (OCT; Figure 1) and fluorescein angiography (FA; Figure 2). His visual symptoms resolved with discontinuation of prednisone, but recurred 5 days after restarting $5 \mathrm{mg}$ prednisone daily for joint pain.

Case report 2. A 65-year-old woman with recurrent anterior uveitis and Addison's disease taking stable doses of azathioprine $75 \mathrm{mg}$ daily and hydrocortisone $30 \mathrm{mg}$ daily complained of blurry vision of the left eye. On examination her vision was counting fingers at 4 feet on the left. She had mild inflammation in the anterior chamber bilaterally. Fundus examination on the left, OCT, and FA revealed a serous macular detachment. Within 3 months, vision improved to 20/50 with clinical resolution of CSR. Three months later she had a recurrence of CSR, which also resolved with time.

Case report 3. A 49-year-old male patient with inflammatory bowel disease, associated arthritis, and sclerokeratitis had a history of bilateral CSR after taking oral corticosteroids. His siblings also had CSR. He received oral steroids prior to a CT scan because of contrast allergy and also received intraarticular steroid injection for herniated disc. Shortly thereafter he noted decreased vision. His vision was 20/50 on the right. Ophthalmic examination revealed serous detachment of the right macula confirmed by OCT and FA. He underwent focal laser photocoagulation to the area of leakage. A month later, vision had improved to 20/30+1 and fundus examination and OCT revealed resolution of CSR.

Case report 4. A 29-year-old male patient with sarcoidosis and uveitis, which had been quiescent for about 18 months, complained of a black spot in his central right vision. Acuity was $20 / 30-2 /+2$ on the right. He had mild inflammation in both anterior chambers. The right macula revealed an area of subretinal fluid and a pigmented epithelial defect superonasal to the fovea with overlying superficial subretinal hemorrhage. Both eyes had peripheral chorioretinal scars. OCT confirmed presence of subretinal fluid and FA revealed leakage in the right eye. He was started on oral prednisone, but 2 weeks later the serous detachment in the macula had enlarged as measured by serial OCT. Oral prednisone was discontinued. Five months after the initial presentation his vision was 20/20 with resolution of CSR as confirmed by OCT.

CSR is a common disorder of the retina characterized by neurosensory detachment of the macula. It is associated with a number of risk factors including type A personality, psychosocial stress, pregnancy, and hypercortisolism ${ }^{1}$. Patients may present with unilateral blurred vision and a variety of visual distortions. It is usually diagnosed clinically with confirmation by FA. Classically, CSR appears as a blister of transparent fluid. On FA the dye leaks from a focal retinal pigment epithelial (RPE) defect and then accumulates within the subretinal space. Visual prognosis especially for acute CSR is good. In most patients there is a resolution within a few months and visual acuity returns to $20 / 25$ or better ${ }^{3}$.

Exogenous administration of steroids and endogenous hypercortisolism have been associated with CSR. Corticosteroids by various routes (including oral, intravenous, intraarticular, epidural, intranasal, inhalational, and topical) have been implicated in $\operatorname{CSR}^{2,4,5,6,7,8}$.

As exemplified in the case reports, corticosteroid use has been associated with the development, recurrence, and worsening of CSR. Caution must be exercised in starting steroids in a patient with a history of CSR, and visual complaints in a patient receiving corticosteroids should prompt an ophthalmologic evaluation. Discontinuation of steroids usually leads to resolution of CSR, but if steroids must be continued and spontaneous recovery does not occur then laser photocoagulation or photodynamic therapy may be considered ${ }^{9,10}$. An awareness of CSR can be a critical component of the decision-making for patients managed jointly by rheumatologists and ophthalmologists.

SEEMA R. GUPTA, MD, Uveitis Fellow, Department of Ophthalmology (Casey Eye Institute), Oregon Health and Science University; ERIC B. SUHLER, MD, MPH, Associate Professor of Ophthalmology, Department

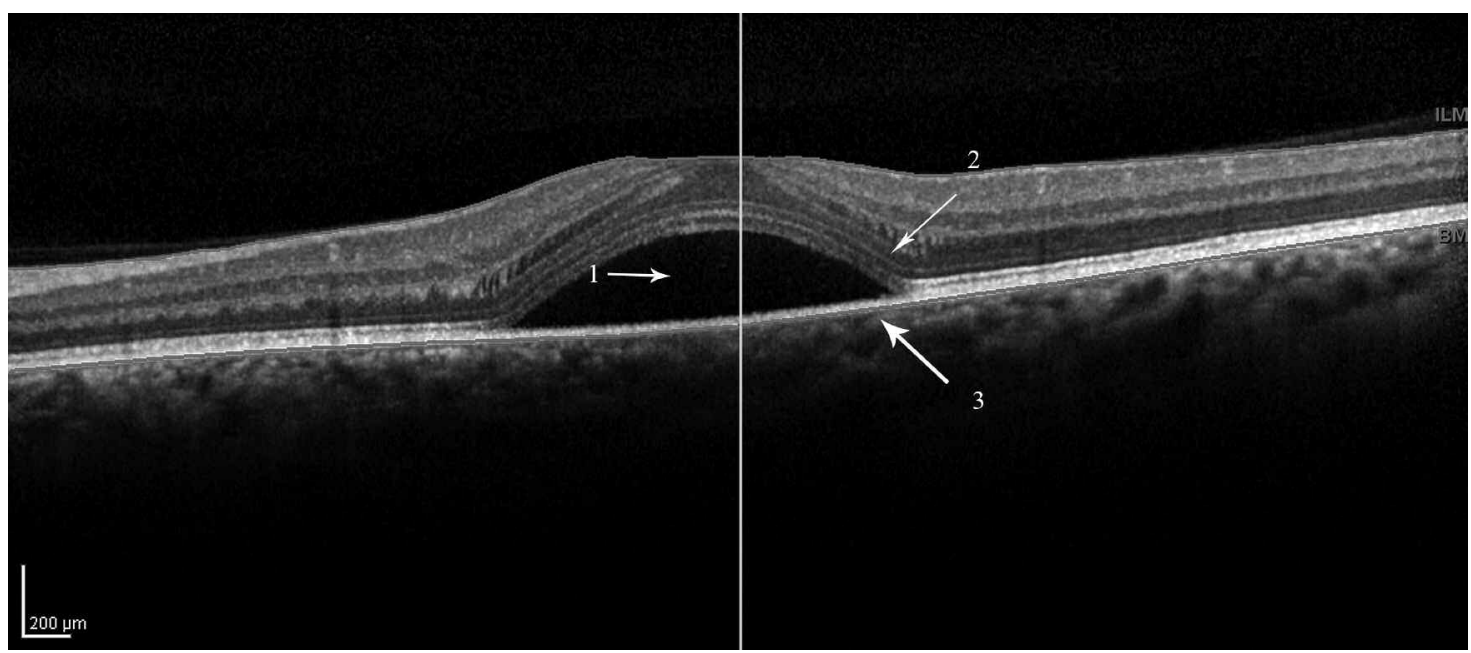

Figure 1. Optical coherence tomography of CSR showing convex-shaped blister of fluid (1) separating the neurosensory retina (2) from retinal pigment epithelium and choroid (3). 


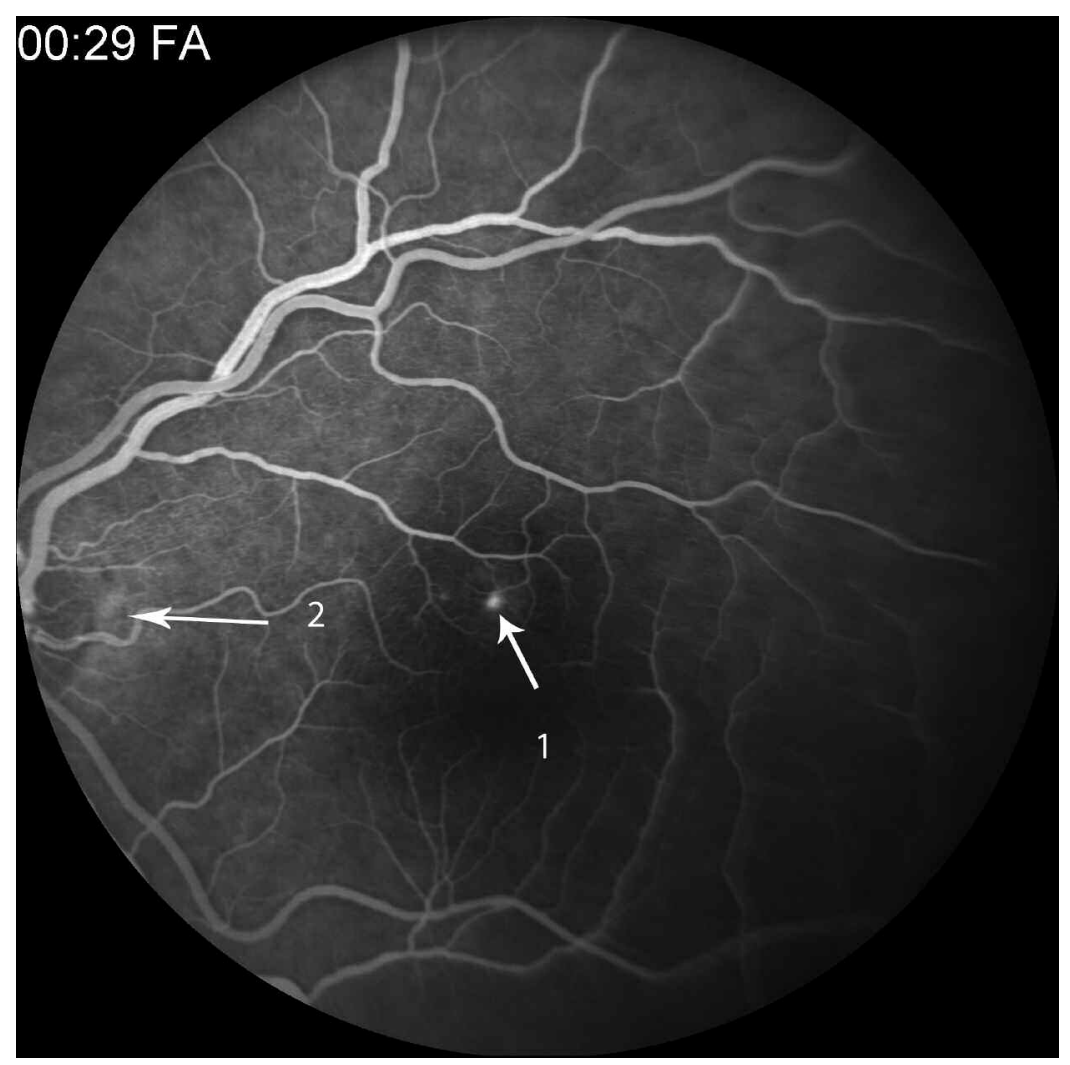

Figure 2. Fluorescein angiography showing focal leakage that appears as a bright hyperfluorescent spot in the macula (1) temporal to the optic disc (2).

of Ophthalmology (Casey Eye Institute), Oregon Health and Science University, Chief of Ophthalmology, Portland, Veterans Affairs Medical Center; JAMES T. ROSENBAUM, MD, Professor of Ophthalmology, Medicine and Cell Biology, Departments of Ophthalmology (Casey Eye Institute), Internal Medicine, and Cell Biology, Oregon Health and Science University. Address correspondence to Dr. S.R Gupta, 3375 SW Terwilliger Blvd., Portland, Oregon 97239;

E-mail: seema_r_gupta@yahoo.com

Supported by the Research to Prevent Blindness to the Casey Eye Institute, Fund for Arthritis and Infectious Disease Research, Stan and Madelle Family Trust, the William and Mary Bauman Foundation, and the US Department of Veterans Affairs.

\section{REFERENCES}

1. Haimovici R, Koh S, Gagnon DR, Lehrfeld T, Wellik S; Central Serous Chorioretinopathy Case-Control Study Group. Risk factors for central serous chorioretinopathy: a case control study. Ophthalmology 2004;111:244-9.

2. Bouzas EA, Karadimas P, Pournaras CJ. Central serous chorioretinopathy and glucocorticoids. Surv Ophthalmol 2002;47:431-48.

3. Klein ML, Van Buskirk EM, Friedman E et al. Experience with nontreatment of central serous choroidopathy. Arch Ophthalmol 1974;91:247-50.
4. Abu el-Asrar AM. Central serous chorioretinopathy complicating systemic corticosteroid therapy. Eur J Ophthalmol 1997;7:297-300.

5. Iida T, Spaide RF, Negrao SG, Carvalho CA, Yannuzzi LA. Central serous chorioretinopathy after epidural corticosteroid injection. Am J Ophthalmol 2001;132:423-5.

6. Mondal LK, Sarkar K, Datta H, Chatterjee PR. Acute bilateral central serous chorioretinopathy following intra-articular injection of corticosteroid. Indian J Ophthalmol 2005;53:132-4.

7. Haimovici R, Gragoudas ES, Duker JS, Sjaarda RN, Eliott D. Central serous chorioretinopathy associated with inhaled or intranasal corticosteroids. Ophthalmology 1997;104:1653-60.

8. Karadimas P, Kapitanios A, Bouzas EA. Central serous chorioretinopathy after local application of glucocorticoids for skin disorders. Arch Ophthalmol 2004;122:784-6.

9. Burumcek E, Mudun A, Karacorlu S, Arslan MO. Laser photocoagulation for persistent central serous retinopathy: results of long-term follow-up. Ophthalmology 1997;104:616-22.

10. Yannuzzi LA, Slakter JS, Gross NE, Spaide RF, Costa DL, Huang $\mathrm{SJ}$, et al. Indocyanine green angiography-guided photodynamic therapy for treatment of chronic central serous chorioretinopathy: a pilot study. Retina 2003;23:288-98.

J Rheumatol 2010;37:9; doi:10.3899/jrheum.091230 